\title{
Cancer et traduction
}

\section{Les nouvelles de ce numéro ont été préparées par Pascale Briand Josette Cadusseau ${ }^{1}$ Hélène Gilgenkrantz ${ }^{2}$ Jean-Claude Dreyfus Jean-Pierre Grünfeld Nathalie Josso ${ }^{3}$ Axel Kahn Brigitte Onteniente ${ }^{4}$ Gérard Orth ${ }^{5}$ Jean-Yves Picard ${ }^{3}$ Guy Riou ${ }^{6}$ Bernard Vigier ${ }^{3}$}

1. Inserm U. 161, 2 rue d'Alésia, 75014 Paris, France.

2. ICGM, Inserm U. 129, 24 rue du Fatubourg Saint-Jacques, 75014 Paris, France. 3. Inserm U. 293, Ecole Normale Supérieure, département de biologie, 1 rue Maurice Arnoux, 92120 Montrouge, France.

4. Inserm U. 161,2 rue d'Alésia, 75014 Paris, France.

5. Inserm U. 190, unité des Papillomavirus, Institut Pasteur. 25-28 rue clu Docteur Roux 75015 Paris, lirance.

6. Laboratoire de pharmacologie clinique et moléculaire. Institut Gustave Roussv, rue Camille Desmoulins. 9.4800 Villejuif, France.
Les ARN messagers eucaryotiques ont un nucléotide 5' lié à une structure spéciale dénommée coiffe, ou cap, qui consiste en un acide guanylique triphosphate méthylé ( ${ }^{m i} \mathrm{GpppX} .$. ). Cette structure doit être reconnue par le facteur d'initiation de la traduction eIF-4, et plus particulièrement par la sous-unité eIF-4E qui est activée par la phosphorylation d'un résidu sérine.

Cette réaction activatrice peut être déclenchée par les agents stimulant les divisions cellulaires. L'équipe de Nahum Sonenberg, un grand spécialiste de la traduction des messagers travaillant à l'université McGill de Montréal (Canada), s'est donc posée la question du rôle d'eIF-4E comme l'un des intermédiaires présomptifs de la cascade d'événements aboutissant au déclenchement du cycle cellulaire. Des cellules en culture ont été infectées par trois types de vecteurs rétroviraux : l'un ne contenait pas la séquence d'ADN codant pour eIF-4E, un autre contenait cette séquence mutée au niveau du codon sérine correspondant au site de phosphorylation, et le troisième contenait la séquence normale. Les cellules exprimant de grandes quantités du facteur eIF-4E normal avaient acquis un phénotype transformé et étaient tumorigènes chez la souris immunodéprimée alors que celles exprimant le facteur muté, ou n'exprimant pas de facteur mais ayant intégré le rétrovirus vecteur, n'étaient absolument pas transformées. Les mécanismes par lesquels une hyper-expression d'un facteur d'initiation de la traduction peut entraîner la cancérisation des cellules sont incertains. Certains messagers d'oncogènes sont très peu traductibles, possédant une longue extrémité 5' non codante semblant jouer un rôle d'inhibiteur de la traduction. Tel est le cas des $A R N m$ des gènes c-sis (codant pour une chaîne du PDGF, platelet derived growth factor), c$m y c$ et lck. Une hypothèse possible est que l'hyperactivité du facteur eIF-4 aboutisse malgré tout à la traduction efficace de ces messagers, ou de messagers jouant des rôles similaires, et par conséquent à l'accumulation de protéines stimulant la prolifération cellulaire. Quoique ce mécanisme reste hypothétique, il n'en reste pas moins que voilà rapporté un niveau de plus dont la perturbation peut provoquer une prolifération incontrôlée. Après des facteurs de transcription, des protéines du cytosquelette, des récepteurs membranaires, des G-protéines, des tyrosine et des sérine kinases, des facteurs de croissance, des molécules d'adhérence et maintenant des facteurs de traduction, on peut se demander, avec un certain sens de la provocation, quels sont les systèmes vraiment indépendant du cycle cellulaire et dont aucune perturbation ne peut entrấner de prolifération anormale ! Sans aller jusque-là, comme nous le soulignions il y a dix-huit mois $\left(\mathrm{m} / \mathrm{s}, n^{\circ} 10\right.$, vol. 4, p. 606), la notion d'oncogène doit sûrement être revue et ne pas englober tous les systèmes potentiellement capables d'activer les mitoses dans des conditions expérimentales particulières, mais doit probablement se limiter aux gènes jouant réellement un rôle dans la progression tumorale "naturelle".

A. $\mathbf{K}$.

1. Lazaris-Karatzas A, Montine KS, Sonenberg N. Malignant transformation by a eukaryotic initiation factor subunit that binds to mRNA 5' cap. Nature $1990 ; 345: 544-7$. 